\title{
Preparation of Nano- and Microstructures For Drug Delivery
}

\author{
Z. Ahmad ${ }^{1,3}$ and M. Edirisinghe ${ }^{2}$
}

Received 9 May 2017; accepted 11 May 2017; published online 2 June 2017

\begin{abstract}
Numerous advances in pharmaceutical sciences and drug delivery have focused on nano- and microstructural aspects of dosage form development. These have been approached through engineering, chemical, and material perspectives and provide numerous opportunities to enhance therapeutic delivery using various administration routes. Progress on these scales has been far reaching; both in terms of application and also stage of formulation development, delivering significant and timely concepts on "miniaturised" scales. This thematic issue provides a broad coverage of developments focusing on numerous aspects of nano- and micron scale structures (properties, materials, and architectures) which may arise from common and emerging preparation methods. The guest editors would like to thank the authors for their contributions which were mainly driven by an EPSRC initiative focusing on emerging pharmaceutical technologies.

The thematic issue includes an example of modification to micro/nano-crystalline acetaminophen through binary polymeric mixtures and ultra-sonication to prepare particles up to $10 \mu \mathrm{m}$ in size (Nokhodchi, 2017, DOI: 10.1208/s12249-016-0596x). A separate study (Elkordy, 2017, DOI: 10.1208/s12249-0160682-0) focuses on the in situ crystallisation of naproxen to yield microparticulate systems in conjunction with freeze-drying techniques. While both of the aforementioned preparation methods have been explored to an appreciable degree, an article focusing on the development of microencapsulated bcarotene via less common zeta-potential yield stress phenomenon is shown (Ghosal, 2017, DOI: 10.1208/s12249-017-0806-1). An emerging jet-based pharmaceutical technology, namely electrohydrodynamic atomisation, is used to prepare microparticles with multiple compartments (in Janus format) hosting multiple model compounds for chemotherapy (Williams, 2017,
\end{abstract}

\footnotetext{
${ }^{1}$ Leicester School of Pharmacy, De Montfort University, Leicester, LE1 9BH, UK.

${ }^{2}$ Department of Mechanical Engineering, University College London, WC1E 7JE, London, UK.

${ }^{3}$ To whom correspondence should be addressed. (e-mail: zahmad@dmu.ac.uk)
}

DOI: 10.1208/s12249-016-0638-4). The process is operational at the ambient environment and, like zeta-potential yield stress phenomena, provides a route for the preparation of sensitive API hosting microparticles. Smaller particles are also of great interest and importance to this theme, especially for applications targeting IV delivery. Using the same technique to prepare microparticles (Nokhodchi et al., 2017, DOI: 10.1208/s12249016-0621-0) uses probe sonication to prepare both solid lipid nanoparticles and nano-structured lipid carriers which display enhanced solubility rates for poorly soluble spironolactone. Also focusing on lipid systems (Cai et al., 2017, DOI: 10.1208/ s12249-016-0637-5) develops and characterises a nano-particle system to treat brain injury through efficient delivery of progesterone. The method they deploy in this instance is emulsion based, and their approach utilises quality-by-design implementation through a step-wise approach. Moving away from particulate systems, Das et al. demonstrate the importance of both material and engineering-based development of microneedles. In the first instance, the use of fish-scale polymer composites delivering lidocaine is shown (Das et al., 2017, DOI: 10.1208/s12249-017-0758-5). Furthermore, the impact of microneedle geometry is also elaborated using Rizatriptan (Das et al., 2017, DOI: 10.1208/s12249-016-0702-0). Both articles relate to importance of microstructure development (using naturally occurring microstructures and bio-polymers) for microneedle-based drug delivery. The final article in this thematic issue focuses on developments in porous materials as potential drug delivery systems (Ahmad et al., 2017, DOI: 10.1208/s12249-017-0740-2). The article provides a detailed review of progress to date with respect to inorganic materials on the micro- and nanometer scales.

Open Access This article is distributed under the terms of the Creative Commons Attribution 4.0 International License (http://creativecommons.org/licenses/by/4.0/), which permits unrestricted use, distribution, and reproduction in any medium, provided you give appropriate credit to the original author(s) and the source, provide a link to the Creative Commons license, and indicate if changes were made. 